\title{
The disulfide bond Cys2724-Cys2774 in the C-terminal cystine knot domain of von Willebrand factor is critical for its dimerization and secretion
}

\author{
Yuxin Zhang ${ }^{1,2}$, Fengwu Chen², Aizhen Yang ${ }^{2}$, Xiaoying Wang ${ }^{1}$, Yue Han ${ }^{2}$, Depei Wu ${ }^{2}$, Yi Wu ${ }^{1,2^{*}}$ and \\ Jingyu Zhang ${ }^{1 *}$
}

\begin{abstract}
Background: Type 3 von Willebrand disease (VWD) exhibits severe hemorrhagic tendency with complicated pathogenesis. The C-terminal cystine knot (CTCK) domain plays an important role in the dimerization and secretion of von Willebrand factor (WWF). The CTCK domain has four intrachain disulfide bonds including Cys2724-Cys2774, Cys2739-Cys2788, Cys2750-Cys2804 and Cys2754-Cys2806, and the single cysteine mutation in Cys2739-Cys2788, Cys2750-Cys2804 and Cys2754-Cys2806 result in type 3 WWD, demonstrating the crucial role of these three disulfide bonds in WWF biosynthesis, however, the role of the remaining disulfide bond Cys2724-Cys2774 remains unclear.

Method and results: In this study, by the next-generation sequencing we found a missense mutation a c.8171G $>A$ (C2724Y) in the CTCK domain of WWF allele in a patient family with type 3 WWD. In vitro, WWF C2724Y protein was expressed normally in HEK-293T cells but did not form a dimer or secrete into cell culture medium, suggesting that C2724 is critical for the WWF dimerization, and thus for WWF multimerization and secretion.

Conclusions: Our findings provide the first genetic evidence for the important role of Cys2724-Cys2774 in VWF biosynthesis and secretion. Therefore, all of the four intrachain disulfide bonds in CTCK monomer contribute to VWF dimerization and secretion.
\end{abstract}

Keywords: von Willebrand factor (VWF), von Willebrand disease (VWD), cystine knot domain, multimerization, disulfide bond

\section{Introduction}

VWF is a large-molecular multimerized glycoprotein synthesized and secreted by endothelial cells and megakaryocytes. This protein has multiple contributions to the hemostatic process: it mediates platelet adhesion and aggregation at sites of vascular injury and carries coagulation factor VIII (FVIII) in the circulation[1]. Functional

\footnotetext{
*Correspondence: yiwu99@gmail.com; zjyhbsjz@163.com

'Department of Hematology, Key Laboratory of Hematology of Hebei

Province, The Second Hospital of Hebei Medical University, 050000 Shijiazhuang, China

Full list of author information is available at the end of the article
}

and/or quantitative deficiencies of VWF are known as von Willebrand disease (VWD), a disorder affecting $0.01-1 \%$ of the population[1]. These patients with VWF deficiency manifest a severe hemorrhagic phenotype, originating from defective formation of platelet-rich thrombi and a secondary deficiency of FVIII impairing the generation of a fibrin network[1]. Quantitative deficiencies of VWF result from changes in biosynthesis, secretion, and/or clearance of the protein.

VWF processor contains multiple domains in the order of D1-D2-D9-D3-A1-A2-A3D4-B1-B2-B3-C1-C2-

C C The Author(s). 2021 Open Access This article is licensed under a Creative Commons Attribution 4.0 International License, which permits use, sharing, adaptation, distribution and reproduction in any medium or format, as long as you give appropriate credit to the original author(s) and the source, provide a link to the Creative Commons licence, and indicate if changes were made. The images or other third party material in this article are included in the article's Creative Commons licence, unless indicated otherwise in a credit line to the material. If material is not included in the article's Creative Commons licence and your intended use is not permitted by statutory regulation or exceeds the permitted use, you will need to obtain permission directly from the copyright holder. To view a copy of this licence, visit http://creativecommons.org/licenses/by/4.0/. The Creative Commons Public Domain Dedication waiver (http://creativecommons.org/publicdomain/zero/1.0/) applies to the data made available in this article, unless otherwise stated in a credit line to the data. 
CK[2]. The VWF sequence contains an unusually high content of cysteine residues (8.3\%), a percentage fourfold higher than the average in human proteins. In the biosynthesis of VWF, its multimerization is a critical step and involves the pairing of cysteines. pro-VWF monomers are linked pairwise through interchain disulfide bond(s) at the C-terminal cystine knot (CTCK) domain[3]. The CTCK domain has 11 cysteines, 8 of which form 4 intrachain disulfide bonds, Cys2724-Cys2774, Cys2739-Cys2788, Cys2750-Cys2804 and Cys2754Cys2806, the other 3 cysteines form 3 interchain disulfide bonds [4] ensuring the long-term stability of the dimeric conformation of VWF. In the endoplasmic reticulum (ER), pro-VWF subunits first engage into a covalent connection, involving the formation of 3 interchain cysteine pairs located in the C-terminal cysteine knot (CK) domains. Importantly, the structural location of these 3 intermolecular pairs protects them from disulfide reduction, ensuring the long-term stability of the dimeric conformation of VWF. Homozygous mutations of C2739Y[5], C2804Y[6], C2754W[7, 8], and C2806R[9] caused type 3 VWD. These mutations impair C-terminal dimerization and consequently, preventing the formation of long multimers, demonstrating that these three disulfide bonds Cys2739-Cys2788, Cys2750-Cys2804, and Cys2754-Cys2806 are critical for VWF biosynthesis. However, the functional role of the remaining disulfide bond of Cys2724-Cys2774 in VWF biosynthesis remains unknown.

In this study, we diagnosed a patient family with type 3 VWD, next-generation sequencing revealed that the proband had a VWF allele with a c.3814delT deletion mutation and the other VWF allele with a c.8171G>A (C2724Y) missense mutation in CK domain. In a cell expression system, we found that the VWF C2724Y mutation did not affect VWF monomer expression but caused defective VWF dimerization and secretion. These data provide the first evidence for the critical role of the disulfide bond Cys2724-Cys2774 in CTCK domain in VWF biosynthesis and secretion.

\section{Materials and methods}

\section{Blood samples and laboratory tests}

This study was approved by the Ethics Committee of the Second Hospital of Hebei Medical University. All patients were informed about the experimental nature of this study and gave their consent to participation. Venous blood collected using $105 \mathrm{mM}$ sodium citrate as anti-coagulant was centrifuged at $4000 \mathrm{~g}$ for $10 \mathrm{~min}$ at room temperature (RT), followed by collection of plasma. Within $2 \mathrm{~h}$ after sample collection, activated partial thromboplastin time (APTT) and FVIII coagulant activities (FVIII:C) were tested by an automatic coagulation instrument (ACLTOP700, Spanish Wolfen Group).
Plasma VWF antigen (VWF:Ag) was measured using an enzyme-linked immunosorbent assay (ELISA) kit according to the manufacturer's instructions (HemosIL ${ }^{\circ}$ von Willebrand Factor Antigen, Spanish Wolfen Group).

\section{VWF multimer analysis}

Analysis of VWF multimers in plasma from patients, cell lysate, and cell cultural medium was performed using $1.5 \%$ SDS-agarose gel electrophoresis, as previously described with slight modification[10, 11]. Briefly, the plasma was diluted 1: 5 and cell lysate and cultural medium were diluted $1: 3$ with sample dilution buffer (0.5 M Tris/HCl, pH 6.8, 0.5 M EDTA, 0.1\% SDS, $9 \mathrm{M}$ Urea) and heated at $60{ }^{\circ} \mathrm{C}$ for 30 minutes. Fifteen $\mu \mathrm{L}$ of sample was loaded and VWF multimer was separated by electrophoresis at $3 \mathrm{~mA}$ for $10 \mathrm{~h}$. The gel was washed with double distilled (dd) $\mathrm{H}_{2} \mathrm{O}$, air-dried and blocked with $5 \%$ skimmed milk. VWF multimers were detected by immunoblotting with a rabbit anti-human VWF antibody (Dako).

\section{Screening for mutations}

Genomic DNA of the blood cells from the proband was screened by next-generation sequencing. Two mutations c.3814delT deletion and c.8171G $>$ A mutation in VWF gene of the proband were discovered, the firstgeneration sequencing was used to verify the mutations. DNA sequences containing the c.3814delT deletion mutation and c.8171G $>$ A mutation in exon 28 and exon 52 of the VWF gene were amplified by polymerase chain reaction (PCR) using the primers of VWF-6059034-F: 5'GGATAGGTATCCGAACACGGAG-3', VWF6,059,034-R: 5'- ACAAGAGGGTTGCTTTAGCCAT-3', VWF-6,128,770-F: 5' - CAGCTCTGACGGTCGCTTC-3', and VWF-6,128,770-R: 5' - TCTGTGGGAATATGGA AGTCATTG-3'. The amplified DNA fragments were sequenced directly.

\section{Plasmid construction}

The plasmid pSVHVWF containing the full-length wildtype human VWF cDNA was kindly provided by Dr. Evan Sadler (Washington University School of Medicine, St Louis, USA). Plasmid of VWF C2724Y was constructed by PCR-based mutagenesis using the PSVHVWF plasmid expressing VWF-WT (wild-type) as a template. The sequence of site-directed mutation primers is 5'- GTGAGG AGCCTGAGTACAACGACATCACTGC-3'. PCR amplification conditions: pre-denaturation at $95^{\circ} \mathrm{C}$ for $30 \mathrm{~s}$; denaturation at $98^{\circ} \mathrm{C}$ for $10 \mathrm{~s}$, annealing at $65^{\circ} \mathrm{C}$ for $30 \mathrm{~s}$, extension at $72^{\circ} \mathrm{C}$ for $10 \mathrm{~min}$, repair extension at $72^{\circ} \mathrm{C}$ for 15 min after 22 cycles. PCR products were digested at $37^{\circ} \mathrm{C}$ by the enzyme of Dpn I for $3 \mathrm{~h}$ and transformed into E. coli $\mathrm{DH} 5 \alpha$ competent cells by the method of heat shock. Plasmids of VWF WT and VWF C2724Y were 
purified with the endotoxin-free plasmid extraction kit from Qiagen, and verified by sequencing.

Expression of recombinant VWF protein in HEK-293T cells HEK-293T cells were cultured in Dulbecco's modified Eagle's medium (DMEM) with high glucose supplemented with $10 \%$ fetal bovine serum. Cells were seeded in 6well plate to reach $50-70 \%$ confluence at transfection. Transient transfection was performed with a total of $2 \mu \mathrm{g}$ of WT or C2427Y mutant VWF plasmid, or mixture of WT and C2427Y mutant plasmids using PolyJet ${ }^{\mathrm{m}}$ transfection reagent according to the manufacturer's instructions (SignaGen ${ }^{\circledR}$ Laboratories). After 48 h, cells and culture supernatant were harvested, and the cells were lysed with a buffer containing $100 \mathrm{mM}$ Tris/ $/ \mathrm{HCl}$, $\mathrm{pH} 7.5,1 \%$ Triton X-100 and proteinase inhibitor cocktail (Sigma). Cell lysate and culture supernatant was concentrated to $1 / 10$ of the starting volume using an ultrafiltration cassette (Millipore), and analyzed for VWF expression and multimerization.

\section{Results}

Diagnosis of a patient family with type 3 VWD

The proband was a 12-year-old girl, suffered frequently from large ecchymosis in bump injury since childhood. She was diagnosed as type 3 VWD for intermittent petechiae and coagulopathy when she was 5 years old. She had a history of admitting to hospital with severe menorrhagia and was treated with transfusion of cryoprecipitated FVIII when she was 11 years old. During the next year, she experienced several times of excessive menstrual bleeding. Neither her parents nor her sister at 20year-old had a bleeding tendency. The results of laboratory tests showed that the proband had a prolonged activated partial thromboplastin time (APTT, $66.8 \mathrm{~s}$ ), low plasma VWF Antigen level (VWF:Ag, 2\%) and markedly reduced FVIII coagulant activities (FVIII:C, 2\%). As shown in Fig. 1, the multimer analysis of plasma VWF showed that the VWF multimer was almost missing in the proband, while VWF from the proband's parents had a normal concentration and multimer distribution. This result explained the abnormal coagulation activity of the plasma from the proband and was consistent with the diagnosis of type 3 VWD.

\section{Identification of candidate VWF gene mutations}

To understand the genetic basis underlying the VWD in this family, we isolated genomic DNA from the blood cells of the proband, with which the next-generation genetic sequencing was performed. Sequencing analysis revealed that the proband had a VWF allele from her mother with a c.3814delT deletion mutation in exon 28, predicting a frameshift-mutations involving the loss of Cys1272; and the other VWF allele from her father had

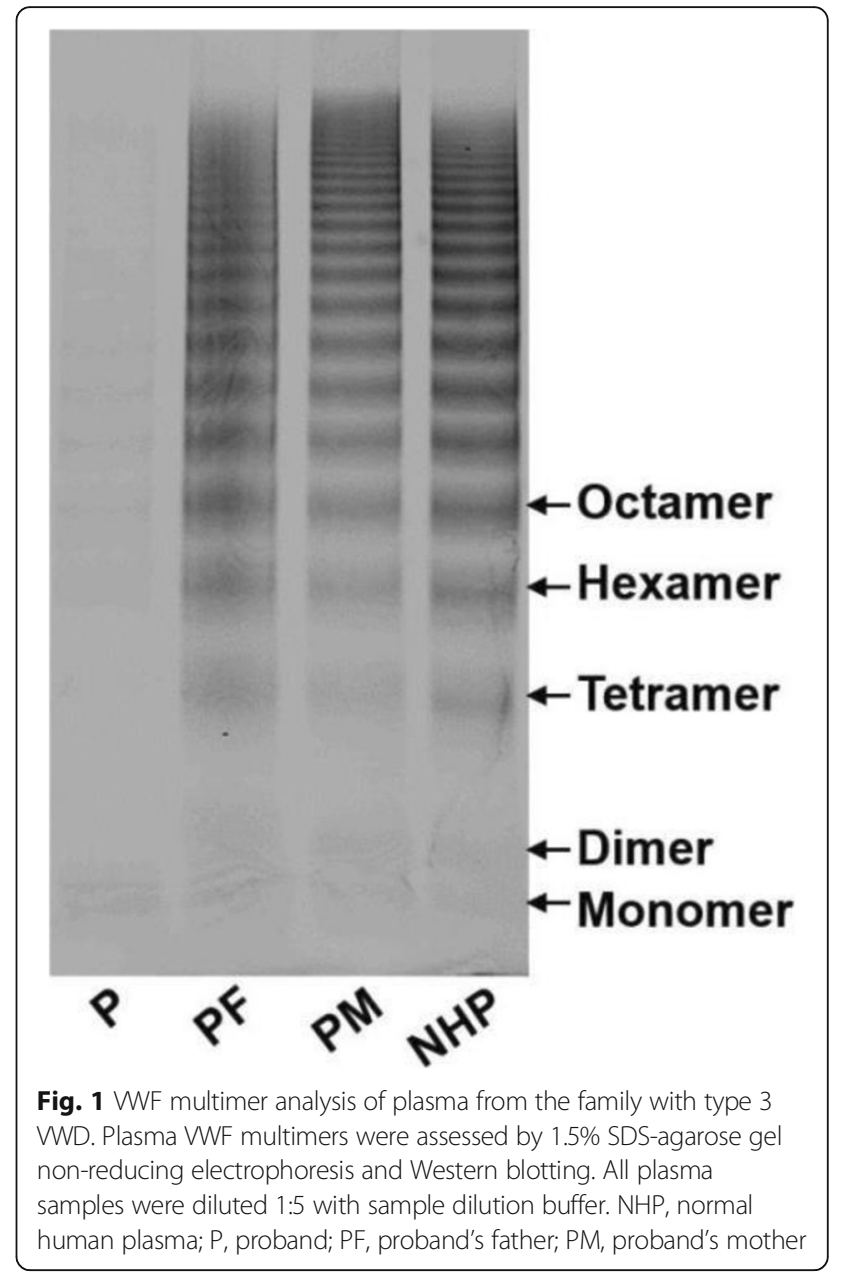

a c.8171G $>$ A missense mutation in exon 51, predicting a substitution of cysteine with tyrosine at amino acid 2724. The accuracy of the results of next-generation genetic sequencing was verified by the first-generation sequencing (Fig. 2 A). Cys2724 locates in the CK domain (Fig. 2B).

\section{Expression of recombinant WT and mutant VWF in mammalian cells}

To determine the mechanism of C2724Y mutation leading to type 3 VWD, we constructed a C2724Y mutant VWF plasmid by PCR-based site-directed mutagenesis. The results of genetic sequencing indicated a successful mutation of Cys2724 in VWF cDNA (Fig. 3 A). To characterize the effect of the C2724Y mutation on VWF synthesis and secretion, HEK-293T cells were transfected with plasmids of VWFWT, VWF-C2724Y, and mixture of VWF-WT and VWF-C2724Y. After $72 \mathrm{~h}$, the transfected cells and culture supernatant was tested for VWF antigen level by reduced SDS-PAGE and western 
A

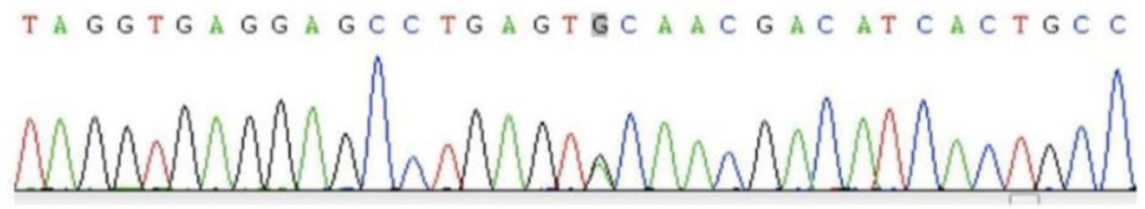

Mutation: exon 51, c.8171G>A:p.C2724Y

B

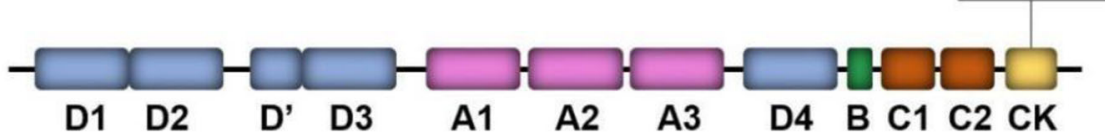

Fig. 2 Identification of candidate WWF gene mutations. A Results of first-generation sequencing showed that one VWF allele of the proband had a c.8171G>A:p.C2724Y mutation in exon 51. B The location of the C2724Y mutation site in the CK domain of WWF molecular

A

\section{VWF G8171A (C2724Y)}

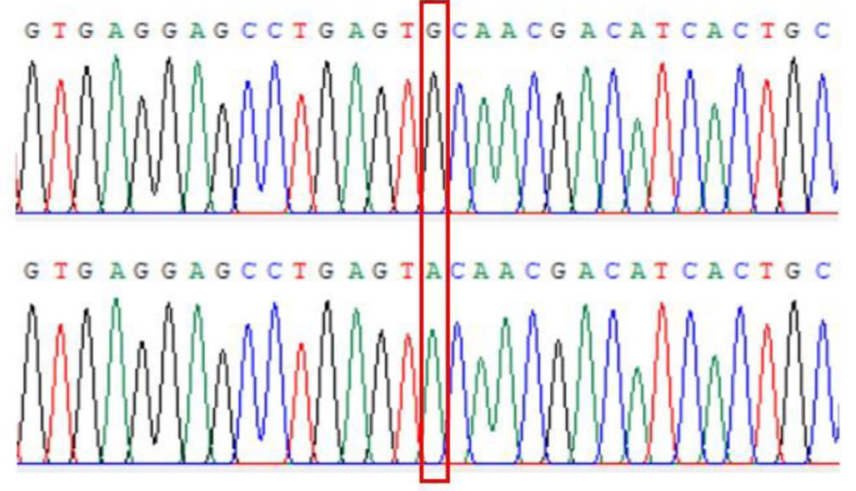

B

Cell lysate

Culture supernatant

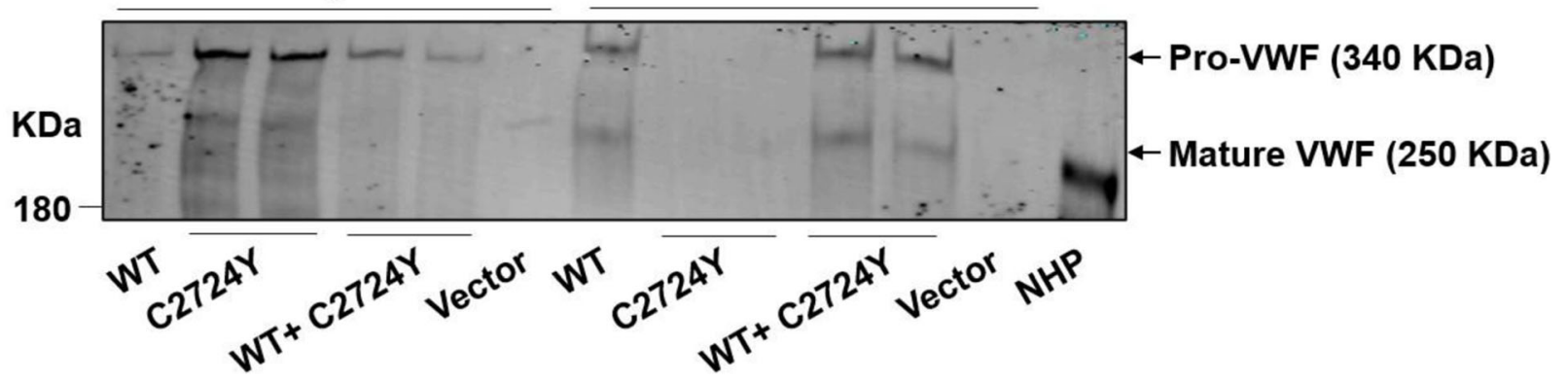

Fig. 3 Expression of recombinant WW protein in HEK-293T cells. A The results of genetic sequencing showed a $\mathrm{G}>\mathrm{A}$ mutation at position 8171 of WWF. B Western blotting analysis of concentrated lysate and supernatant from cultured cells transfected with the plasmids of WWF WT, WWF C2724Y, and mixture of WWF WT and WF C2724Y. Protein in the lysate and supernatant was separated by 8\% reducing SDS-PAGE and immunoblotted with anti-WWF antibody 
blotting. As shown in Fig. 3B, recombinant VWF C2724Y protein was expressed normally in HEK-293T cells, but it could not be secreted into cell culture medium, compared with VWF-WT protein. This result, which is consistent with the reduced VWF antigen level in plasma of the proband with the C2724Y mutant, suggests that $\mathrm{C} 2724-\mathrm{C} 2774$ disulfide bond is critical for the VWF secretion.

\section{Multimer analysis of recombinant VWF-C2724Y protein expressed in HEK293T cells}

To further determine whether C2724Y mutation affects VWF multimerization, we analyzed the multimer pattern of the lysate and culture supernatant of cells transfected with the plasmid of VWF-WT, VWF-C2724Y, and mixture of VWF-WT and VWFC2724Y. As shown in Fig. 4 A, both recombinant VWF-WT and VWFC2724Y were expressed in HEK-293T cell, however, dimerized product was observed only with the recombinant VWF-WT, while VWF-C2724Y only had a single band of monomer. In cell culture supernatant, there was no expression of VWF C2724Y, although normal expression and multimer pattern of VWF WT and coexpression of VWF WT and VWF C2724Y were detected (Fig. 4-B), consistent with the immunoblotting in the reduced condition. These results indicated that mutation of C2724Y causes defective VWF dimerization, which further leads to dysfunction of VWF multimerization and secretion.

\section{Discussion}

Cys2724 has been reported to form a disulfide bond with Cys2774. In this study, we found a new type 3 VWD family with the proband having almost no VWF antigen and markedly reduced activity of VWF:RCo and FVIII:C $(<5 \%)$ [12]. The proband experienced several times of severe menorrhagia, had a prolonged APTT, low level of plasma VWF Antigen (2\%), and markedly reduced FVIII coagulant activities (2\%). The proband had a VWF allele with a c.3814delT deletion mutation in exon 28 , and the other VWF allele with a c.8171G>A missense mutation in exon 51. The latter mutation causes a substitution of cysteine 2724 to tyrosine. These data provide new evidence revealing the critical role of the disulfide bond Cys2724-Cys2774 in VWF biosynthesis.

There are 11 cysteines contained in VWF CK domain, 8 of them form 4 intrachain disulfide bonds, Cys2724-Cys2774, Cys2739-Cys2788, Cys2750-Cys2804, and Cys2754-Cys2806, the other 3 cysteines form 3 interchain disulfide bonds, Cys2771- Cys2773', Cys2771'-Cys2773, and Cys2811Cys2811'[4]. Mutations of C2739Y[5], C2754W[7, 8], C2804Y[6], and C2806R[9] have also been proven to cause type 3 VWD phenotype, indicating a critical role of disulfide bonds Cys2739-Cys2788, Cys2750-Cys2804, and Cys2754-

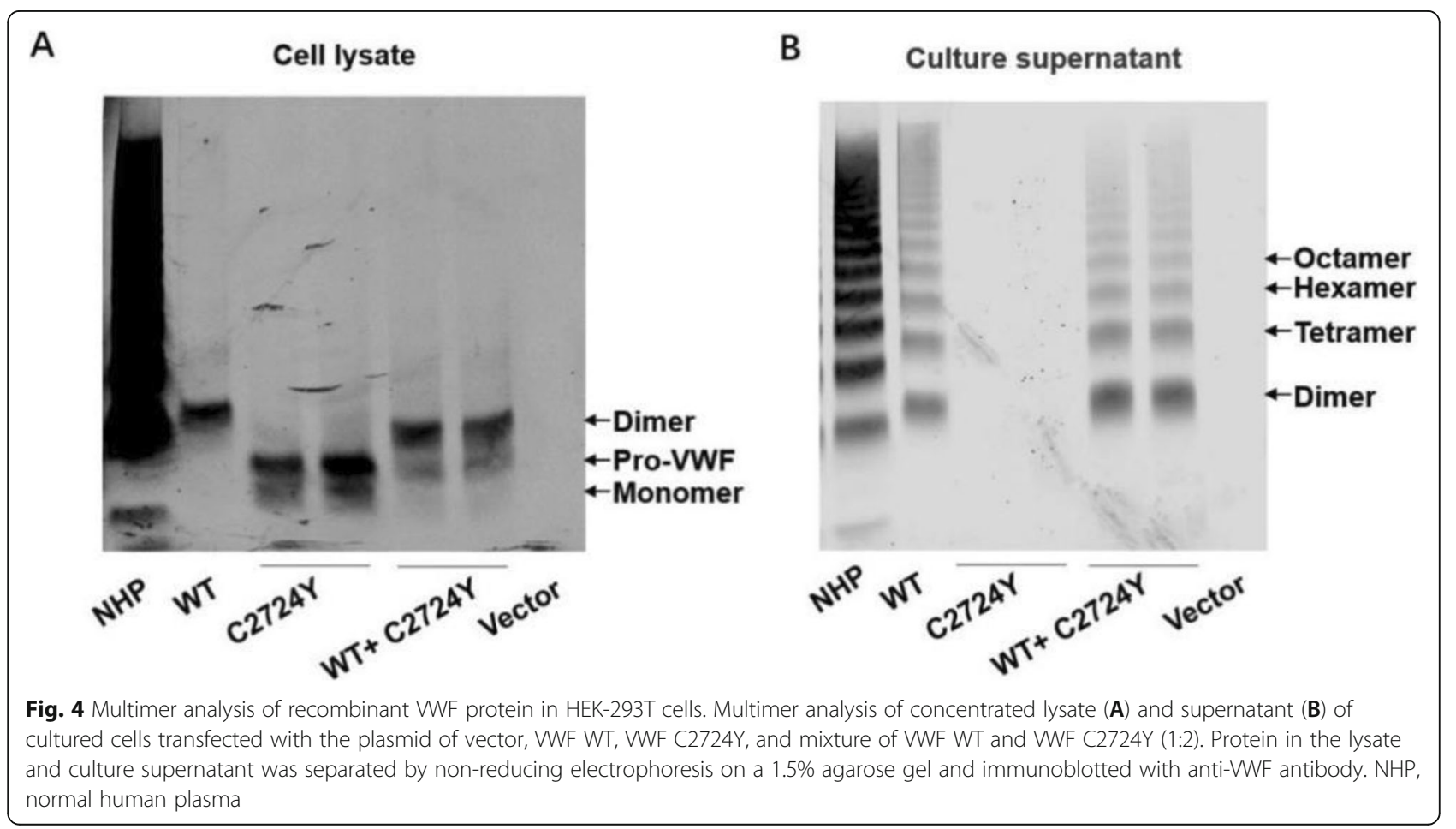


Table 1 Mutations of disulfide bond in VWF CK domain reported in the literatures

\begin{tabular}{lllll}
\hline & Disulfide bond & Mutation & Phenotype & Reference \\
\hline Intrachain & Cys2724-Cys2774 & C2724Y & Type 3 WWD & This paper \\
& Cys2739-Csy2788 & C2739Y & Type 3 WWD & {$[5]$} \\
& Cys2750-Csy2804 & C2804Y & Type 3 WWD & {$[6]$} \\
& Cys2754-Cys2806 & C2754W, C2806R & Type 3 WWD & {$[7-9]$} \\
Interchain & Cys2771-Cys2773' & C2771S/C2771Y, C2773S & Type 2 A/IID WWD & {$[13-15]$} \\
& Cys2771'-Cys2773 & C2771S/C2771Y, C2773S & Type 2 A/IID WWD & {$[13-15]$} \\
& Cys2811-Cys2811' & C2811A & Normal WWF multimerization & and secretion, but occurrence \\
& & & of odd-numbered multimer
\end{tabular}

Cys2806 in VWF multimerization and secretion. Compared with the mutations of cysteines forming intrachain disulfide bonds, the mutations of cysteines in interchain disulfide bonds in CK domains did not cause serious phenotypes. Homozygous mutations of C2771S/C2771Y[13] and C2773S $[14,15]$ did not affect secretion of VWF protein, manifested as type 2 A VWD, subtype IID (VWD 2 A/IID). Mutation of Cys2811 affects neither VWF dimerization nor VWF secretion, but leads to the occurrence of odd-numbered multimer[15-17] (Table 1). These results indicate that the intrachain disulfide bonds in CK domain are much more important than the interchain disulfide bonds in VWF dimerization and secretion. Up to now, mutations of VWF Cys2750, Cys2774, or Cys2788 have not yet been reported.

Cys2724 locates at the N-terminal of VWF CK domain, forms an intrachain disulfide bond with Cys2774[4]. In this study, we provided the evidence showing that the disruption of this Cys2724-CysC2774 caused defective VWF multimerization and secretion in cells, a potential new mechanism underlying the pathogenesis of type 3 VWD. To determine the mechanism of C2724Y mutation leading to type 3 VWD, we constructed a VWF-C2724Y plasmid, then transfected plasmid of VWF-WT, VWF-C2724Y, or together into HEK-293T cells, and analyzed the expression and multimerization of recombinant VWF in transfected cells and cultural supernatant. The results showed that recombinant VWF-C2724Y protein expressed normally in HEK-293T cells, however, it cannot form a dimer or secrete into cell culture medium, while VWF-WT protein expressed and secreted normally, as well had a normal multimer pattern (Fig. 3B). We transfected the plasmids into Hela cells and get the same results (data not shown). These results indicated that mutation of $\mathrm{C} 2724 \mathrm{Y}$ causes defective VWF dimerization, which further leads to defective VWF multimerization and secretion, which interprets the reduction of VWF antigen level in plasma from the proband. Based on our new finding of the mutation of C2724Y in new family of type 3 VWD, all of the four intrachain disulfide bonds (Cys2724-Cys2774, Cys2739-Cys2788, Cys2750-Cys2804, and Cys2754Cys2806) in CK monomer are critical for VWF dimerization and secretion, the disruption of any of these disulfide bonds may lead to the significant reduction of VWF antigen in plasma and development of type 3 VWD.

Although Cys2754, Cys2773, Cys2739, and Cys2724 are cysteines of interchain disulfide bonds in CK domains, and mutations of them disrupt the dimerization of VWF and result in abnormal multimerization, however, there are odd-numbered multimers in plasma from patients with heterozygous C2754W[7, 8] and C2773S[14, 15] mutations, as well as the medium of co-expression of VWF WT and C2754W or C2773S protein, while the plasma from patients with heterozygous C2724Y, and medium of co-expression of VWF WT and C2739Y[18] or C2724Y protein had no odd-numbered multimers. The underlying mechanisms remain further study. As shown in the VWF multimer analysis of cell lysate, VWF with C2739Y mutation cannot form a dimer and exist in the form of proVWF(Fig. $4 \mathrm{~A}$ ), supporting that the dimer formation of pro-VWF through the N-terminus in Golgi apparatus is dependent on the formation of CK-linked dimer in ER[19].

\section{Conclusions}

In summary, we have identified a novel missense C2724Y mutation in VWF CK domain in a family of patients with type 3 VWD. This C2724Y mutation caused defective VWF dimerization and secretion, providing the first evidence showing critical role of the disulfide bond Cys2724-Cys2774 in VWF biosynthesis.

\section{Acknowledgements \\ Not applicable.}

\section{Authors' contributions}

$J Z$ and YW conceived the project and designed the experiments; YZ, FC, AY and $X W$ performed the experiments and analyzed the data with supervision from JZ and YW; YH and DW assisted with experimental reagents and interpretation of the data; FC, YW and JZ wrote the manuscript. All authors have read and approved the final manuscript.

\section{Funding}

This work was supported by grants from the National Natural Science Foundation of China $(81770138,81970128,31970890)$, the Translational Research Grant of NCRCH (2020ZKPA02, 2020WSA04), the collaboration fund from State Key Laboratory of Radiation Medicine and Protection 
(GZN1201802), the Priority Academic Program Development of Jiangsu Higher Education Institutions, Hebei Provincial Central Guiding Local Science and Technology Development Fund Project (Basic Research Project) (216Z7705G), and 2020 Hebei Provincial Government Funded Clinical Medical Talents Training Project (Academic Leader) (303-16-20-14).

\section{Availability of data and materials}

The datasets during and/or analysed during the current study available from the corresponding author on reasonable request.

\section{Declarations}

\section{Ethics approval and consent to participate}

This study was approved by the Ethics Committee of the Second Hospital of Hebei Medical University. All patients were informed about the experimental nature of this study and gave their consent to participation.

\section{Consent for publication}

Consent for publication have been obtained from the patients.

\section{Competing interests}

The authors declare that they have no competing interests.

\section{Conflict of interest}

The authors declare no conflicts of interest.

\section{Author details}

'Department of Hematology, Key Laboratory of Hematology of Hebei Province, The Second Hospital of Hebei Medical University, 050000 Shijiazhuang, China. ${ }^{2}$ National Clinical Research Center for Hematologic Diseases, the First Affiliated Hospital, Collaborative Innovation Center of Hematology, State Key Laboratory of Radiation Medicine and Prevention, Cyrus Tang Medical Institute, Soochow University, 215123 Suzhou, China.

Received: 28 September 2021 Accepted: 17 November 2021

Published online: 27 November 2021

\section{References}

1. Lenting PJ, Christophe OD, Denis CV. von Willebrand factor biosynthesis, secretion, and clearance: connecting the far ends. Blood. 2015;125:2019-28.

2. Voorberg J, Fontijn R, van Mourik JA, Pannekoek H. Domains involved in multimer assembly of von willebrand factor (VWF): multimerization is independent of dimerization. EMBO J. 1990;9:797-803.

3. Katsumi A, Tuley EA, Bodo I, Sadler JE. Localization of disulfide bonds in the cystine knot domain of human von Willebrand factor. J Biol Chem. 2000; 275:25585-94.

4. Zhou YF, Springer TA. Highly reinforced structure of a C-terminal dimerization domain in von Willebrand factor. Blood. 2014;123:1785-93.

5. Zhang ZP, Blomback M, Egberg N, Falk G, Anvret M. Characterization of the von Willebrand factor gene (WWF) in von Willebrand disease type III patients from 24 families of Swedish and Finnish origin. Genomics. 1994;21:188-93.

6. Baronciani L, Cozzi G, Canciani MT, Peyvandi F, Srivastava A, Federici AB, Mannucci PM. Molecular characterization of a multiethnic group of 21 patients with type 3 von Willebrand disease. Thromb Haemost. 2000;84: 536-40.

7. Schneppenheim R, Budde U, Obser T, Brassard J, Mainusch K, Ruggeri ZM, Schneppenheim S, Schwaab R, Oldenburg J. Expression and characterization of von Willebrand factor dimerization defects in different types of von Willebrand disease. Blood. 2001;97:2059-66.

8. Tjernberg P, Vos HL, Castaman G, Bertina RM, Eikenboom JC. Dimerization and multimerization defects of von Willebrand factor due to mutated cysteine residues. J Thromb Haemost. 2004;2:257-65.

9. Montgomery RR, Jozwiak MA, Hutter JJ, Endres JL, Foster PA, Friedman KD. A homozygous variant of von Willebrand factor (VWF) that fails to Cterminal dimerize resulting in loss of VWF multimers larger than dimer. Blood. 1999;94:443a-443a.

10. Chen J, Hinckley JD, Haberichter S, Jacobi P, Montgomery R, Flood VH, Wong R, Interlandi G, Chung DW, Lopez JA, Di Paola J. Variable content of von Willebrand factor mutant monomer drives the phenotypic variability in a family with von Willebrand disease. Blood. 2015;126:262-9.
11. Chen J, Hobbs WE, Le J, Lenting PJ, de Groot PG, Lopez JA. The rate of hemolysis in sickle cell disease correlates with the quantity of active von Willebrand factor in the plasma. Blood. 2011;117:3680-3.

12. Branchford BR, Di Paola J. Making a diagnosis of WWD. Hematology Am Soc Hematol Educ Program. 2012;2012:161-7.

13. Enayat MS, Guilliatt AM, Surdhar GK, Jenkins PV, Pasi KJ, Toh CH, Williams MD, Hill FGH. Aberrant dimerization of von Willebrand factor as the result of mutations in the carboxy-terminal region: identification of 3 mutations in members of 3 different families with type 2A (phenotype IID) von Willebrand disease. Blood. 2001;98:674-80.

14. Schneppenheim R, Brassard J, Krey S, Budde U, Kunicki TJ, Holmberg L, Ware J, Ruggeri ZM. Defective dimerization of von Willebrand factor subunits due to a Cys-> Arg mutation in type IID von Willebrand disease. Proc Natl Acad Sci U S A. 1996;93:3581-6.

15. Tjernberg P, Vos HL, Spaargaren-van Riel CC, Luken BM, Voorberg J, Bertina RM, Eikenboom JC. Differential effects of the loss of intrachain- versus interchain-disulfide bonds in the cystine-knot domain of von Willebrand factor on the clinical phenotype of von Willebrand disease. Thromb Haemost. 2006;96:717-24.

16. Lippok S, Kolsek K, Lof A, Eggert D, Vanderlinden W, Muller JP, Konig G, Obser T, Rohrs K, Schneppenheim S, et al. von Willebrand factor is dimerized by protein disulfide isomerase. Blood. 2016;127:1183-91.

17. Lof A, Konig G, Schneppenheim S, Schneppenheim R, Benoit M, Budde U, Muller JP, Brehm MA. Advancing multimer analysis of von Willebrand factor by single-molecule AFM imaging. PLoS One. 2019;14:e0210963.

18. Wang JW, Valentijn KM, de Boer HC, Dirven RJ, van Zonneveld AJ, Koster AJ, Voorberg J, Reitsma PH, Eikenboom J. Intracellular storage and regulated secretion of von Willebrand factor in quantitative von Willebrand disease. J Biol Chem. 2011;286:24180-8.

19. Leebeek FW, Eikenboom JC. Von Willebrand's Disease. N Engl J Med. 2016; 375:2067-80.

\section{Publisher's Note}

Springer Nature remains neutral with regard to jurisdictional claims in published maps and institutional affiliations.

Ready to submit your research? Choose BMC and benefit from:

- fast, convenient online submission

- thorough peer review by experienced researchers in your field

- rapid publication on acceptance

- support for research data, including large and complex data types

- gold Open Access which fosters wider collaboration and increased citations

- maximum visibility for your research: over $100 \mathrm{M}$ website views per year

At BMC, research is always in progress.

Learn more biomedcentral.com/submissions 